Recepción: 18 / 04 / 2018

Aceptación: 26 / 05 / 2018

Publicación: 02 / 07 / 2018

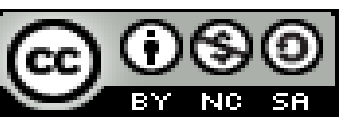

Ciencias técnicas y aplicadas

Artículo de Investigación

\title{
Plan de manejo integral de residuos sólidos orgánicos como alternativa de prevención a la salud
}

Comprehensive management plan for organic solid waste as an alternative to health prevention

Plano de gestão abrangente de resíduos sólidos orgânicos como alternativa à prevenção da saúde

\author{
Juan R. Cedeño-Candela ${ }^{\mathrm{I}}$ \\ jcandela_24@hotmail.com \\ Marcos R. Gallo-Zambrano II \\ arqmarcosg@hotmail.com
}

Correspondencia: jcandela_24@hotmail.com

\footnotetext{
I Magister en Gestión Ambiental, Arquitecto, Docente de la Universidad Laica Eloy Alfaro de Manabí, Manta, Ecuador.

II Magister en Gestión Ambiental, Magister en Arquitectura Mención Diseño Urbano, Arquitecto, Docente de la Universidad Laica Eloy Alfaro de Manabí, Manta, Ecuador.
} 


\title{
Resumen
}

Se establece una propuesta de gestión integral de residuos sólidos orgánicos en albergues temporales del cantón Pedernales, armonizando la protección de la integridad física y la salud humana en dichos asentamientos con aspectos medioambientales y urbanísticos para prevenir y/o mitigar impactos posteriores al terremoto del 16 de abril de 2016. La investigación fue elaborada con un enfoque cuantitativo y a partir de un paradigma empírico - analítico racionalista. Para el desarrollo de la misma se emplearon los siguientes métodos: histórico (tendencial), hipotético deductivo, así como el de medición. Los resultados de la medición, permitieron determinar la generación per cápita de residuos sólidos y su clasificación por tipos en los albergues estudiados. Entre las conclusiones, se comprobó que no se realiza un manejo adecuado de residuos sólidos en los albergues "Divino Niño" y "Los Llanos" del cantón Pedernales. Para ello se propone un plan de manejo integral de residuos sólidos orgánicos como alternativa de prevención a la salud.

Palabras clave: plan; residuos sólidos; residuos orgánicos; prevención a la salud.

\begin{abstract}
A proposal for integral management of organic solid waste is established in temporary shelters of the canton of Pedernales, harmonizing the protection of physical integrity and human health in these settlements with environmental and urban aspects to prevent and / or mitigate impacts after the earthquake of December 16. April 2016. The research was elaborated with a quantitative approach and based on an empirical-analytical rationalist paradigm. For the development of the same the following methods were used: historical (trend), hypothetical deductive, as well as the measurement. The results of the measurement, allowed to determine the per capita generation of solid waste and its classification by types in the shelters studied. Among the conclusions, it was found that an adequate management of solid waste is not carried out in the "Divino Niño" and "Los Llanos" shelters of the Pedernales district. To this end, a comprehensive management plan for organic solid waste is proposed as an alternative to health prevention.
\end{abstract}

Keywords: plan; solid waste; organic waste; prevention to health.

\section{Resumo}

Uma proposta de gestão integrada dos resíduos sólidos orgânicos em abrigos temporários Pedernales Canton set, harmonizar a protecção da integridade física e à saúde humana em tais 
acordos com os aspectos ambientais e espaciais para evitar e / ou mitigar os impactos subseqüentes terremoto 16 Abril de 2016. A pesquisa foi elaborada com uma abordagem quantitativa e baseada em um paradigma racionalista empírico-analítico. Para o desenvolvimento dos mesmos foram utilizados os seguintes métodos: histórico (tendência), hipotético dedutivo, bem como a medida. Os resultados da medição permitiram determinar a geração per capita de resíduos sólidos e sua classificação por tipos nos abrigos estudados. Entre as conclusões, constatou-se que a gestão adequada dos resíduos sólidos não é realizada nos abrigos "Divino Niño" e "Los Llanos" do distrito de Pedernales. Para tanto, propõe-se um plano abrangente de manejo de resíduos sólidos orgânicos como alternativa à prevenção da saúde.

Palavras chave: plano; resíduos sólidos; resíduos orgânicos; prevenção à saúde.

\section{Introducción}

El género humano se ve cada vez más afectado por el incremento de la frecuencia de los desastres relacionados con el clima. En los últimos 20 años, se cuentan en varios miles de millones la cantidad de heridos y damnificados por estos fenómenos. En ese mismo período, los desastres de carácter geofísico han provocado más muertes que todos los otros peligros naturales combinados (CRED, 2015). Además de pérdidas de vidas humanas, traen como consecuencia afectaciones materiales a las personas y el traslado de estas a recintos seguros y albergues temporales. Para calificar a estas personas se utiliza el término de desplazados internos. El ser humano, al realizar sus actividades de tipo industrial, comercial y doméstico genera productos de desecho, cuyos volúmenes totales por habitante habitualmente aumentan en el tiempo.

Mención aparte merecen los desastres de carácter geofísico. En las últimas dos décadas, los terremotos representaron más muertes que todos los otros peligros naturales combinados, causando casi el 56\% de la mortalidad total por desastres en el mundo entre 1996 y 2015. En este sentido, se plantea el hecho que debido a las características geológicas que posee y a la actividad antropológica del territorio nacional del Ecuador, haciéndolo sensible a deslizamientos de tierra y procesos erosivos, sobre todo cuando aparecen persistentes lluvias.

Esto ha provocado que, sólo como consecuencia de los principales eventos naturales ocurridos en el país en los últimos sesenta años, la cifra de damnificados sobrepasa los 3'400.000 personas. Y a pesar que en el contexto nacional la responsabilidad de la prevención, reducción y 
resarcimiento de las consecuencias de los siniestros, ha pasado de la Dirección Nacional de Defensa Civil a la Secretaría Técnica de Gestión de Riesgos, la respuesta gubernamental ante estos embates ha sido, básicamente, de carácter reactivo, centrando las prioridades en las acciones ulteriores a la ocurrencia del desastre para situaciones de emergencia y, en concreto, en las tareas de recuperación y restauración de hogares y servicios básicos.

Bajo este escenario, la gestión de residuos sólidos no ha sido tenida en cuenta en toda su magnitud en los albergues en los que se han visto obligados a vivir temporalmente los miles de seres humanos que se cuentan entre los desplazados internos, tal es el caso del evento sísmico, registrado el 16 de abril de 2016 en la costa ecuatoriana y que afectó fundamentalmente a las provincias del litoral y en específico a Manabí y a Esmeraldas, dejó un saldo de 671 fallecidos y 69.000 edificaciones afectadas. La magnitud de este siniestro fue tal que entre 70.000 y 80.000 personas se convirtieron en desplazados internos.

El gobierno hubo de instaurar un total de 26 albergues oficiales en Manabí y en Esmeraldas. En dichos enclaves se situaron alrededor de 12.000 albergados tras el sismo. Tres meses después de producirse el evento, aún se encontraban albergadas más de 8.600 personas. Sólo luego de un año, se logró cerrar el último albergue oficial administrado por el gobierno.

Los sitios escogidos para fungir como albergues, no siempre estaban predefinidos. Varios de ellos fueron elegidos de modo apresurado y en ocasiones improvisando. Uno de los procesos a los que no se les prestó toda la atención requerida fue la gestión de residuos sólidos que se había de aplicar en los albergues. Para complementar esta actividad se empleó prácticamente el mismo régimen que se utiliza a nivel de la sociedad y que dista, en buena medida, de ser un modelo apropiado. A tal efecto, el artículo refiere a los albergues "Divino Niño" y "Los Llanos 2", en la jurisdicción del cantón Pedernales, los cuales se localizan en los límites urbanos del cantón y fueron construidos por el cuerpo de ingenieros del ejército de Ecuador en mayo de 2016, a partir de una decisión del gobierno nacional para acoger a más de 200 familias cada uno, para ello se propuso como objetivo el establecimiento de una propuesta de gestión integral de residuos sólidos orgánicos en albergues temporales del cantón Pedernales, armonizando la protección de la integridad física y la salud humana en dichos asentamientos con aspectos medioambientales y urbanísticos. 


\section{Desarrollo}

\section{Residuos sólidos}

Los residuos sólidos se definen por todo objeto, sustancia o elemento en estado sólido, que se abandona bota o rechaza (Glyn, 1991). Por su parte, el Diario oficial de las Comunidades Europeas (1991), define los residuos como "toda sustancia o todo objeto, del que el poseedor se desprende o de la que tiene la intención o la obligación de deshacerse"(p.45). También se conceptualizan como tal, aquellos materiales cuyo poseedor desecha y que pueden ser susceptibles de recibir tratamiento o disposición final de conformidad con lo que establecen las regulaciones ambientales de cada país o región (Wehenpohl, Hernández, Rodríguez y Escudero, 2006).

Los residuos sólidos se pueden clasificar de diversos modos, según su origen, nivel de peligrosidad, características físicas, químicas o biológicas, entre otras.

De acuerdo a su origen, los residuos pueden ser industriales, derivados de procesos constructivos, hospitalarios y domésticos. Habitualmente, el origen de los residuos establece las peculiaridades físicas, químicas y biológicas que poseen y, en consecuencia, su peligrosidad y tratamiento.

Concretamente son considerados residuos urbanos aquellos que producen los habitantes de una ciudad o población, no sólo como residuo propio, sino también, como consecuencia de las actividades que en dichas ciudades tienen lugar. Esta definición incluye los residuos típicos generados en un núcleo urbano; domésticos, viarios, comerciales, de mercado, industriales y sanitarios.

\section{Gestión integral de residuos sólidos}

La gestión integral de residuos sólidos urbanos está formada por actividades dirigidas a controlar las acciones de generar, separar, presentar, almacenar, recolectar, transportar, barrer, aprovechar, tratar y disponer finalmente estos residuos. Estas actividades deben estar relacionadas con el cumplimiento de los mejores principios del ornato público, la ingeniería, la economía, la salud pública y respondiendo a las expectativas de la sociedad. 
Siguiendo lo planteado en el Congreso de la Unión de Estados Mexicanos celebrado en 2003, la gestión integral de residuos sólidos es el conjunto articulado e interrelacionado de acciones normativas, operativas, financieras, de planeación, administrativas, sociales, educativas, de monitoreo, supervisión y evaluación, para el manejo de residuos, desde su generación hasta la disposición final, a fin de lograr beneficios ambientales, la optimización económica de su manejo y su aceptación social, respondiendo a las necesidades y circunstancias de cada localidad o región.

Como se aprecia, en dichas definiciones, existe una intención de tratar los componentes de este sistema dirigidos no solamente hacia los residuos en sí, más bien se intenta hacer trascender los mismos evitando afectaciones a la salud, procurando beneficios ambientales, tratando de lograr la aceptación ciudadana, pues sin esta no se garantiza la sostenibilidad, y además adaptada al contexto en el que se desarrolla esta actividad.

\section{Componentes de la gestión integral de residuos sólidos}

Para garantizar el correcto desempeño de un sistema de gestión integral de residuos sólidos, explica Abreu, Rodríguez, Pérez y González (2008), se deben integrar los siguientes componentes: Almacenamiento de residuos, Servicio de recolección, Servicio de barrido, Transporte y Disposición final.

Además, en dependencia del contexto de cada región, deben incluirse los siguientes componentes: Minimización de la generación de recursos, Separación de residuos en la fuente y Colocación (presentación) de los residuos en los recipientes apropiados en los horarios y días establecidos, Recolección diferenciada de residuos, Métodos que permitan la reducción de costos de transportación, Implementación del reúso, separación y reciclaje de los residuos, Tratamiento de residuos orgánicos, Implementación de un relleno sanitario responsable y acorde a las normativas legales establecidas.

Estos componentes deben estar vinculados con las siguientes acciones: reglamentos que garanticen la sostenibilidad y operatividad del sistema, recuperación de costos, optimización operativa de servicios, planificación y administración del sistema e incorporación de la comunidad. 


\section{Jerarquía de la gestión integral de residuos sólidos}

Un sistema de gestión integral de residuos sólidos se debe plantear en base a ciertos principios jerárquicos, asociados a los componentes de este sistema y que tratan de beneficiar a la salud pública y evitar la contaminación ambiental, ante el creciente aumento de los volúmenes de desechos.

El orden de jerarquía pretende establecer lineamientos que deben ser pautas en el papel que deben jugar los diferentes actores inmersos en este proceso. Todo ello, según la Sociedad Nacional de la Cruz Roja Colombiana (2008), debe ser tratado en función de la realidad de la región y a partir de un análisis de las potencialidades existentes.

Este orden jerárquico está establecido en el siguiente orden: Evitar la generación de innecesaria de residuos sólidos, Minimizar la generación de residuos sólidos, Recuperar todo lo posible de lo generado, Procesar y tratar, todo aquello que pueda tener una utilidad y Colocar en un relleno sanitario los desechos que no pueden ser procesados, ni tratados.

\section{Metodología}

La investigación tiene un alcance descriptivo, pues detalla el proceso de manejo de residuos sólidos en los albergues para desplazados internos del cantón Pedernales posterior al terremoto del 16 de abril de 2016, así como busca especificar las características que debe poseer un lugar dado para fungir como recinto para dar cobija a afectados por calamidades naturales y, en consecuencia, medir esta poli-dependiente cualidad. La población considerada es de 1.113 albergados, distribuidos en 283 familias y ocupando 298 carpas de los albergues temporales "Divino niño" acoge a un total de 494 personas y el el albergue "Los Llanos" de Pedernales II con 619 personas. La obtención de los datos e información se obtuvo a través de la aplicación de encuestas y cuestionarios los cuales fueron procesados considerando los siguientes métodos: histórico (tendencial), hipotético deductivo, así como el de medición. En la selección de los criterios para la evaluación de albergues desde una perspectiva medioambiental y urbanística se utilizó el método Delphi propuesto por Landeta (1999), en la búsqueda de consenso y técnicas de evaluación multicriterio para conocer el peso relativo de cada uno de los indicadores. 


\section{Resultados}

Posterior a la recolección de los datos e información a través de la aplicación de encuestas y cuestionarios, se presentan los resultados de sus análisis considerando los métodos: histórico (tendencial), hipotético deductivo, tal como se presentan a continuación.

Tabla 1. Residuos totales por día en cada albergue

\begin{tabular}{|c|c|}
\hline Albergue temporal & $\begin{array}{c}\text { Cantidad de residuos sólidos } \\
\text { estimados [kg/día] }\end{array}$ \\
\hline "Los Llanos" de Pedernales II & 163 \\
\hline "Divino niño" & 130 \\
\hline
\end{tabular}

Elaborado: El autor

Se pudo constatar, que entre ambos albergues se generan $293 \mathrm{~kg} / \mathrm{día}$ de residuos sólidos.

En la figura 1, se muestra los resultados de la clasificación física de los residuos sólidos, en la que se indica que, en el proceso de clasificación de los residuos sólidos, se comprueba que la composición fundamental de los mismos consiste en restos de alimentos, que abarcan más del 50\% de la masa total. Otros componentes como: plásticos, pañales desechables y papel y cartón aparecen, en ambos recintos, en cantidades alrededor del $10 \%$. Por su parte, el caucho, cuero, tierra y metales se encuentran en cuantías despreciables. Todo esto se observa en la figura 1.

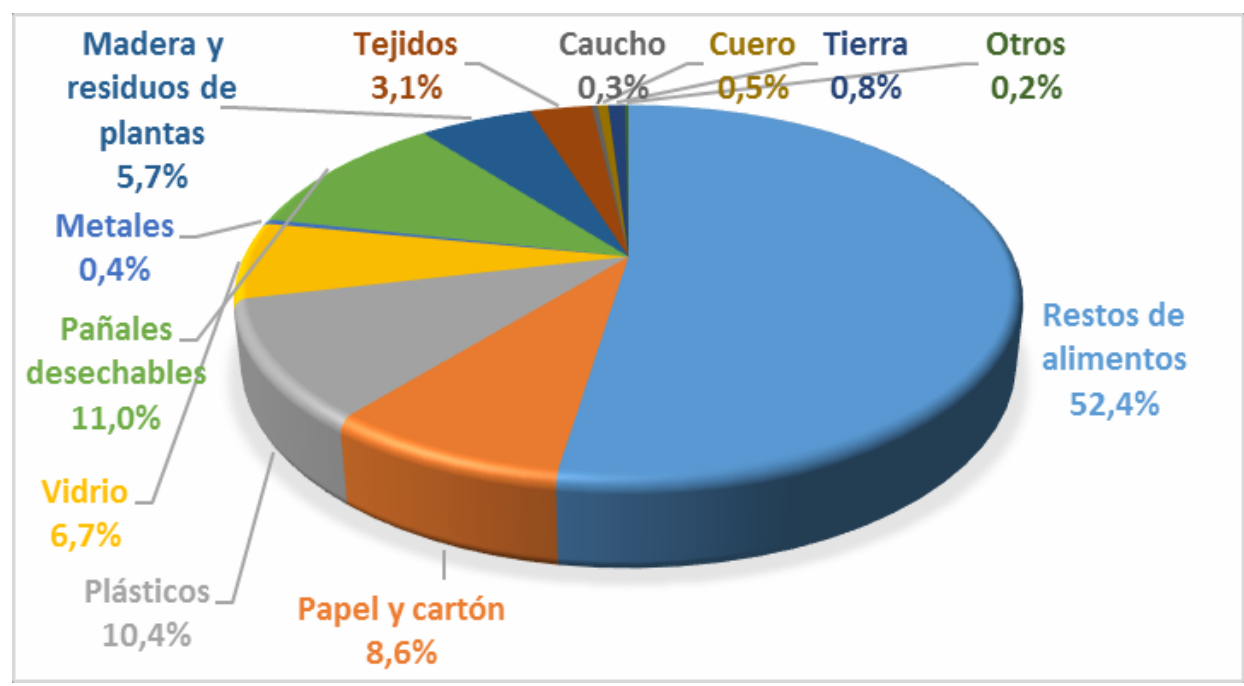

Figura 1. Composición porcentual de los residuos sólidos en los albergues del cantón Pedernales.

Fuente: El autor, 2017 
En la figura 2, se muestra el análisis de los resultados de la densidad de los residuos, en la que se observa que existe una gran similitud en los valores obtenidos para ambos recintos. La densidad media ponderada de los residuos sólidos alcanza el valor de $290,4 \mathrm{~kg} \cdot \mathrm{m}^{\wedge}(-3)$ (ver figura 2).

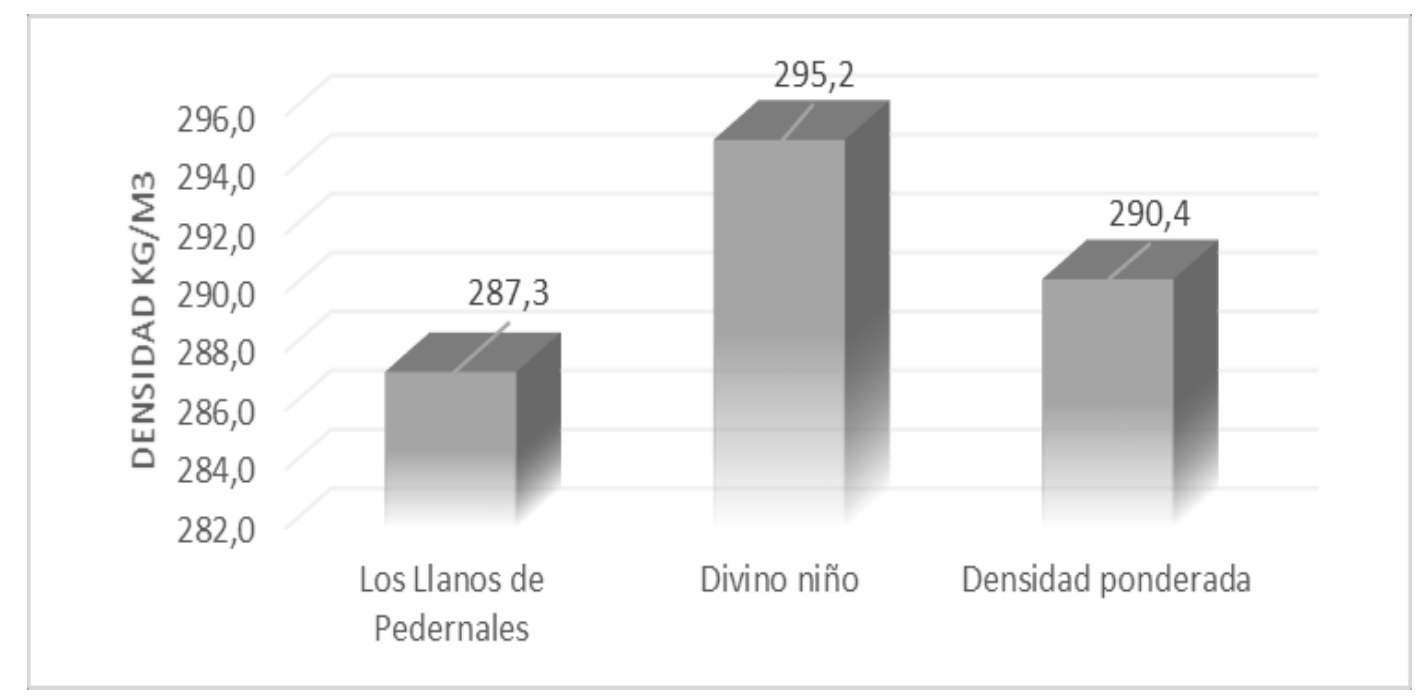

Figura 2. Densidad media de los residuos sólidos en los albergues del cantón Pedernales y densidad media ponderada.

Fuente: El autor, 2017

El impacto más notable que se produce es el aspecto referente a la proliferación de vectores que generan las actividades de manejo de residuos sólidos, el cual se calificó como muy significativo para "Los Llanos" y significativo para "Divino Niño". Los impactos: emisiones de malos olores y contaminación por polvo aparecen -para ambos recintos- calificados como medianamente significativos. Las actividades que más contribuyen con el impacto ambiental son las de almacenamiento y generación de residuales sólidos, llegando a ser responsables en ambos recintos de más de las cuatro quintas partes del impacto ambiental calculado.

Como resultado de la aplicación de la herramienta de evaluación multicriterio, se obtuvo que las variables fundamentales tenidas en cuenta para consignar el desempeño de los albergues quedan categorizadas entre los niveles 1 (sitio poco apropiado) y 2 (sitio apropiado).

El aspecto más vulnerable en el albergue "Divino Niño", fueron las afectaciones debido al clima, mientras que en "Los Llanos" de Pedernales el parámetro de mayor detrimento fue su Ubicación, adoptando en ambos casos la calificación de poco apropiado. 
Si se sintetiza el análisis y se hace una valoración del conjunto, considerando los factores de ponderación de cada variable, se llega a la conclusión que ninguno de los albergues alcanza ni siquiera la categoría de sitio apropiado, tal y como se muestra en la figura 3

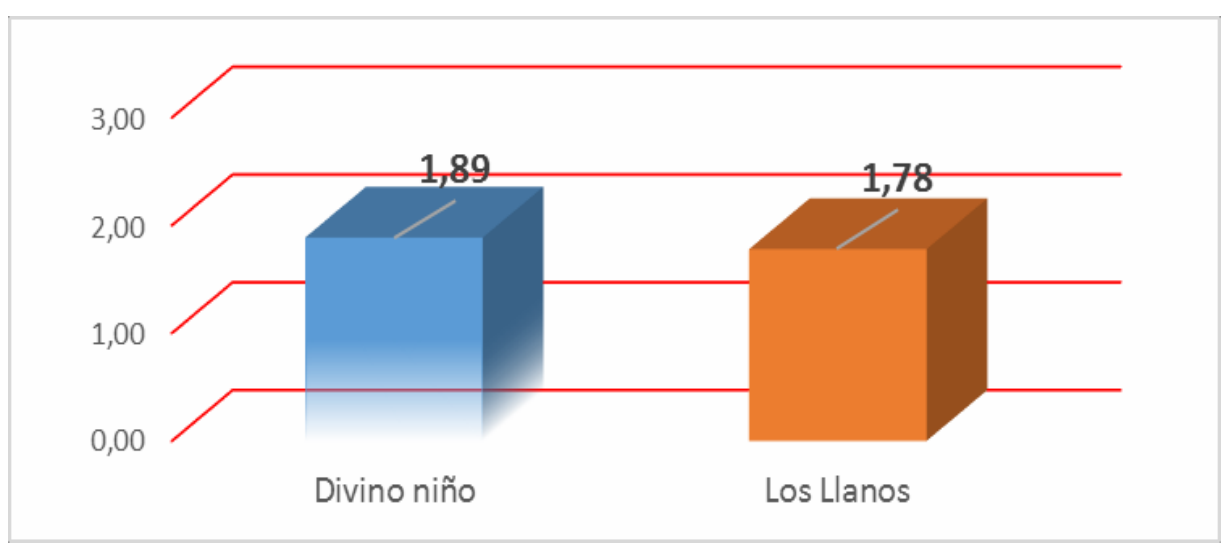

Figura 3. Resultados de la calificación general, aplicando la metodología para la evaluación multicriterio, en los albergues del cantón Pedernales.

Fuente: El autor, 2017

\section{Conclusiones}

Se comprobó que la gestión de los residuos sólidos se enfoca como situación concerniente al servicio, sin tener en cuenta sus componentes ambientales, sociales, económicos y de salud, en la que existe ausencia de un programa de educación ambiental dirigido a la población albergada, manifiesto en la falta de condiciones para el almacenamiento de los residuos, tanto en las carpas como en los sitios de recogida.

Se observó que los desplazados internos poseen poca educación ambiental, principalmente en lo que se refiere a residuos sólidos, ya que más del 50\% de los encuestados no diferenciaban los residuos orgánicos de los inorgánicos, sólo la tercera parte los clasifica en la fuente, pero la mayoría no realiza tareas de reciclaje y sólo la cuarta parte de ellos ha recibido alguna actividad de capacitación en esta área.

La producción per cápita de residuos obtenida para la muestra fue de $\mathbf{0 , 2 6 4} \frac{\mathbf{k g}}{\mathbf{h a b} \cdot \text { día }}$. El valor de producción de residuos es relativamente bajo, en comparación con la generación per cápita promedio en viviendas, cuestión razonable dado el régimen particular en que se desarrolla la vida diaria de los albergados y que son personas que, en muchos casos, han perdido buena parte de su 
patrimonio personal y la desviación estándar de la producción per cápita de residuos obtenida

para la muestra de $171 \frac{g}{h a b \text {-día }}$. Este valor resulta inferior al asumido para la estimación del tamaño de la muestra y les otorga validez práctica a los resultados obtenidos.

La proliferación de vectores se califica como un impacto altamente significativo para "Los Llanos" y significativo para "Divino Niño". Los impactos: emisiones de malos olores y contaminación por polvo aparecen -para ambos recintos- calificados como medianamente significativos.

Las actividades que más contribuyen con el impacto ambiental son las de almacenamiento y generación de residuales sólidos, llegando a ser responsables - en ambos recintos- de más de las cuatro quintas partes del impacto ambiental calculado.

Los dos recintos empleados como tal, no logran cumplir con la categoría de sitios apropiados para fungir como refugio temporal de desplazados internos, atendiendo a deficiencias detectadas en su desempeño ante situaciones climáticas adversas y en cuanto a su ubicación.

Del diagnóstico, se concluye que no se realizan manejos adecuados de residuos sólidos en los albergues "Divino Niño" y "Los Llanos" del cantón Pedernales, por lo que se recomienda aplicar un plan de manejo integral de residuos sólidos orgánicos como alternativa de prevención a la salud.

\section{Justificación de la propuesta}

Las emergencias y desastres ocasionados principalmente por inundaciones, terremotos y vientos huracanados, originan gran cantidad de damnificados, la mayoría de los cuales corresponden a familias de escasos recursos, con pérdidas de sus pertenencias y hogares. Generalmente no existen lugares adecuados para recibir a los afectados, siendo los centros educacionales y comunales los que cuentan con mejores condiciones de infraestructura para estos fines, debido a que son construcciones cerradas, que habitualmente están compuestas por: salones, cocina, comedor, baños, servicios sanitarios y espacios de esparcimiento para una estadía temporal. Sin embargo, emplear estos sitios para dichos fines, implicaría prescindir de los beneficios de la función social para los que originalmente fueron concebidos. Esta situación conlleva a la 
generación otros problemas de carácter urbanístico, asociados a la reubicación funcional de estos espacios citadinos.

En ese sentido, se justifica la selección y planificación de determinadas áreas que - dada una situación de desastre- tengan las condiciones apropiadas en cuanto a: seguridad, ubicación y condiciones de vida para fungir como albergues para desplazados internos durante el tiempo que se requiera y que, en situaciones comunes, desempeñen otras funciones dentro del esquema urbanístico de una región.

\section{Fundamentación}

Una vez establecidos los albergues, los principales problemas que se presentan en su habilitación y funcionamiento en situaciones de emergencias son: Hacinamiento, falta de privacidad, elaboración de alimentos, abastecimiento de agua, eliminación de excretas y residuos sólidos, inactividad (laboral), afectaciones climáticas, recreación y violencia social.

Como se puede apreciar, uno de los inconvenientes que aparecen en albergues temporales es el manejo y disposición adecuada de los desechos sólidos. Como se sabe, los desechos sólidos, si no son tratados convenientemente, se pueden transformar en un factor crítico para la salud pública y en especial en las condiciones en que se ven necesitadas de convivir estas agrupaciones humanas.

Los desechos sólidos si no son debidamente manejados pueden originar importantes problemas de salud y un medio ambiente desagradable para vivir en él, si no se eliminan de manera segura y apropiada. De igual forma, pueden servir de criaderos de insectos, parásitos y de otros animales dañinos, lo cual aumenta la posibilidad de la transmisión de enfermedades. Además, los desechos sin ningún manejo también pueden contaminar las fuentes de agua y el medio ambiente.

\section{Objetivos}

El objetivo de la propuesta es diseñar un conjunto de medidas enfiladas al manejo de los residuos sólidos orgánicos en los albergues de desplazados internos del cantón Pedernales y que serán aplicadas a las actividades de recolección y transferencia, de estos residuos. 


\section{Importancia}

La investigación ahonda en el funcionamiento de los albergues para desplazados internos que se ubicaron en el cantón Pedernales, sobre todo en lo concerniente al modo en que se realiza en ellos actualmente la gestión de residuos sólidos.

Adicionalmente a ello, la propuesta contiene las directivas que podrían aplicar las organizaciones encargadas de establecer las políticas de protección a damnificados debido a fenómenos naturales en cuanto a buenas prácticas que han de seguirse, tanto en la selección de un sitio como albergue temporal, como en la gestión de residuos sólidos en estos espacios.

A esto se suma que ayudará a atenuar tres importantes cuestiones, pues:

- Permite calificar los sitios que se emplean como albergues temporales, atendiendo a una evaluación multicriterio en función de su ubicación, afectación debido a indicadores climáticos, así como a la seguridad y el confort (en cuanto a los servicios y espacios) que brindan a los desplazados internos.

- Incluye la planificación preliminar de un espacio urbano que, ante una situación de desastre natural, reunirá las condiciones necesarias para emplazar allí un albergue temporal, lo cual le dará un mayor nivel de definición a los planes de evacuación diseñados por las autoridades de la defensa civil.

- Mediante las investigaciones de campo se disponen de datos fidedignos y asentados sobre un fundamento estadístico, que contribuyen a sentar las bases para los proyectos de gestión de residuos sólidos en los albergues temporales.

\section{Ubicación sectorial}

La propuesta se desarrolla en la zona suburbana del cantón Pedernales. El área polivalente se localiza al noreste del cantón, justo en la intersección del camino vecinal que lleva al poblado Casa Blanca y la vía al Carmen. Este último acceso vial, constituye precisamente el enlace con el cantón Pedernales. El centro urbano del cantón y el área seleccionada distan en sólo unos 3700 $\mathrm{m}$, lo que garantiza una razonable cercanía a los servicios vitales y de seguridad. Una vista panorámica de la ubicación relativa del área seleccionada se muestra en la figura 4. 


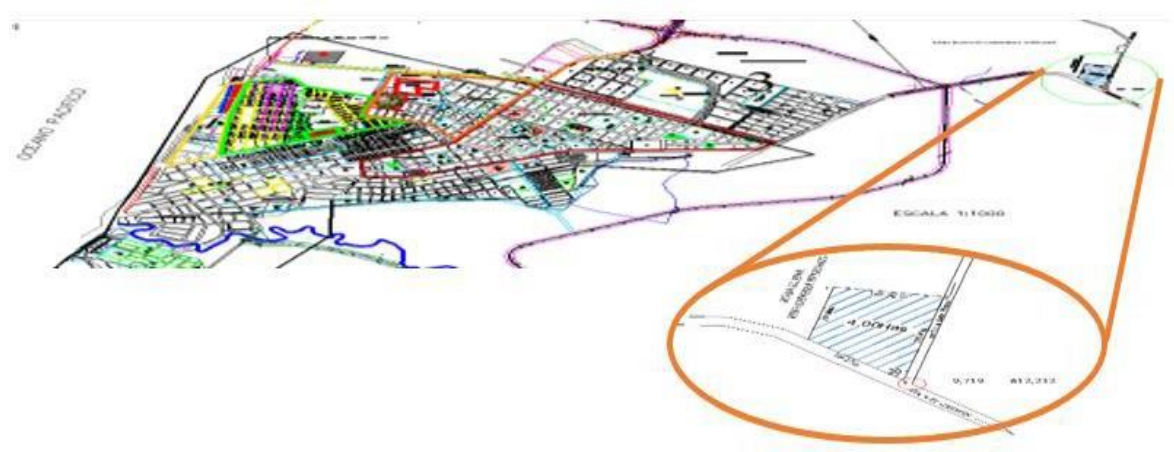

Figura 4. Ubicación del área seleccionada para el Programa de creación de un área urbana que pueda emplearse tanto como área deportiva y albergue temporal

\section{Factibilidad}

La factibilidad de este proyecto parte de la existencia de la ingente necesidad de planear escenarios para la ubicación de desplazados internos. Ante el efecto que traen los fenómenos naturales sobre las comunidades humanas más vulnerables, surge el deber social de crear espacios apropiados para la ubicación temporal de desplazados internos. Estos espacios deben planificarse con antelación y con una visión global del contexto, teniendo presente la interacción de distintos criterios, tales como la ubicación, seguridad, clima, disponibilidad de áreas y la preservación de la integridad física de las personas que las ocupen.

El proyecto es técnicamente viable, pues considera acciones que ejecutivamente no son complejas, así como otras de educación sobre cultura ambiental a los albergados, la creación de condiciones para realizar la clasificación de los residuos en el origen y en el proceso de almacenamiento de estos residuos.

La propuesta es además legalmente viable por las razones expuestas en 2.4

Desde el punto de vista social y ambiental presenta las siguientes ventajas:

- Si se emplean los residuos sólidos orgánicos como compostaje y se clasifican para su comercialización los residuos que pueden ser recuperados, se podría disminuir en un $75 \%$ la masa de residuos sólidos que son llevados a la disposición final 
- Permitirá contar con un espacio que reúne las propiedades necesarias para que los allí albergados disfruten de mejores condiciones de salud y de vida en general

Desde el punto de vista financiero, se pueden obtener ingresos mensuales estimados de casi 1200 USD, durante el tiempo que dure la estancia en el albergue. Este valor se justifica en la tabla XX.

Tabla 2. Ingreso mensual estimado por la venta de productos recuperados

\begin{tabular}{|c|c|}
\hline Producto recuperado & Ingreso estimado mensual(USD) \\
\hline Papel y cartón & 272,45 \\
\hline Plásticos & 461,26 \\
\hline Vidrio & 424,51 \\
\hline Chatarra & 7,60 \\
\hline Total & $\mathbf{1 1 6 5 , 8 2}$ \\
\hline
\end{tabular}

A esto se suman las probadas ventajas desde el punto de vista ambiental (minimización de residuos y recuperación de recursos naturales) que tiene situar fondos hacia la gestión de residuos sólidos.

\section{Descripción de la propuesta}

El plan de gestión de residuos orgánicos presenta un grupo de medidas que permiten controlar y minimizar los efectos desfavorables que las acciones de generación, recolección, transporte, tratamiento y disposición pueden generar.

\section{Programa de capacitación y educación ambiental}

La sensibilización ambiental se considera como un modo de reorientar comportamientos sociales y extender las posibilidades para lograr una mejor calidad de vida en los albergues de desplazados internos.

La educación de los albergados es un aspecto esencial para cambiar el punto de vista acerca de los residuos, sus valores intrínsecos y el modo más apropiado y responsable de desprenderse de ellos. Estas actividades deben tener un carácter permanente. Por su importancia, este aspecto es considerado como un programa. 
Se debe preparar material orientativo y diseñado para llegar a personas de cualquier edad y de los diferentes sectores económicos presentes en el albergue. Estos materiales deben contener mensajes que puedan difundirse de modo claro y comprensible. Además, con estos mensajes se busca sensibilizar y motivar a la población en actividades relacionadas con el reciclaje y el reúso.

De esta manera se trata de empoderar y comprometer a los albergados en que la apropiada gestión de los residuales no es una labor exclusiva de las entidades administrativas y de gobierno.

La participación de los albergados no incluirá solamente su papel en la separación de los residuos en las carpas, como parte de las acciones que conforman el programa, sino que deberán estar informados sobre los detalles de la puesta en funcionamiento del proceso de gestión integral de residuos.

Dentro de esas tareas se encuentran: conocer los objetivos de la separación de residuos, saber que existen diversos procedimientos para el tratamiento de residuales y que estos se encuentran en constante desarrollo, tener idea acerca de las formas en que se comercializan los diferentes tipos de residuos, dominar los beneficios ambientales y económicos del reciclaje de residuos.

La divulgación de estas informaciones puede hacerse mediante carteles, volantes, plegables. También mediante la presencia de las organizaciones de obras públicas y encargadas de estas tareas en las actividades de corte social que se organicen, donde pueden proyectarse vídeos u otros materiales didácticos acerca de los avances, que en estos sentidos se logren, lo que contribuirá a animar a la población albergada para participar de un modo más enérgico en el programa.

Debe prestarse especial atención y darle importancia al hecho de que la separación de los residuos en el núcleo familiar es el primer paso para lograr un cambio de conciencia a nivel de sociedad que permitirá darle continuidad y sistematicidad a los programas de reciclaje.

\section{Programa de separación de residuos sólidos en el origen}

La separación de los residuos debe realizarse en las propias carpas. Los albergados deben ejecutar una clasificación de los mismos, teniendo presente su tipología y el recipiente en el que este se colocará. 
Los tachos se diferenciarán por colores, de acuerdo al tipo de residuo que contendrán. Deben tener asideros para su manejo, poseer tapa hermética, han de ser duraderos y resistentes.

Se propone que los tachos tengan una capacidad de 15 L. Estos se diferenciarán por colores. Los residuos orgánicos se colocarán en depósitos de color verde. El cartón, el papel y los plásticos se han de colocar en depósitos de color amarillo. Mientras tanto, los tachos de color azul serán destinados a los metales y vidrios.

Dada la mínima cuantía de desechos peligrosos que se generan en los albergues no se prevén depósitos para ello. En el caso puntual de las baterías, se recomienda que se destinen recipientes de plástico con tapa con vistas a que ahí sean almacenadas. Para no incurrir en gastos adicionales, se aconseja el uso de pomos plásticos resistentes y reutilizables. Estos recipientes pueden ser de distintas capacidades, desde $0,5 \mathrm{~L}$.

\section{Programa de higiene interior del albergue}

Las familias han de colocar sus desechos en tachos públicos, los cuales deben estar identificados por su color, coincidiendo con el de los domiciliarios. Estos deben ser preferentemente de metal o de plástico de alta resistencia, pues pueden ser sometidos a impactos y fuertes golpes, por parte de la población o los propios recolectores.

El espacio donde se colocan los recipientes para almacenar los residuos, previos a su recolección, deben reunir las siguientes peculiaridades:

- Ubicada en un lugar de fácil localización y acceso por parte de la comunidad albergada y del personal encargado de la recolección.

- Preferentemente, en un espacio limitado por una cerca, para así evitar el acceso de niños y animales.

- Área destinada exclusivamente para este fin, suficientemente alejada de otras zonas de convivencia y convenientemente orientada en función de los vientos predominantes, para evitar que los malos olores puedan originar molestias a la comunidad albergada o a la comunidad.

- El piso, sobre el que se colocan los tachos, debe estar compactado y nivelado. 
- Área techada, evitando la acción directa del sol y la lluvia.

La limpieza de las áreas comunes del albergue, debe realizarse por parte de un obrero, que debe laborar al menos 6 horas diarias. La labor tendrá un carácter manual y debe incluir un set de herramientas, que estaría compuesto por: Escobas (de fibra fibra y plástica), pala, recogedor de basura, carretilla e indumentaria personal básica, compuesta por: uniforme de tela de mezclilla y franjas protectoras, guantes de cuero para las manos, calzado de seguridad, protección respiratoria y visual, casco protector, faja protectora y ponchos para lluvia con capucha.

\section{Programa de creación de un área urbana que pueda emplearse como área deportiva y como albergue temporal}

En todas las actividades que desarrolla el ser humano, se debe considerar que el deporte -aparte de ser una recreación- es una necesidad para mantener una salud equilibrada.

Las ciudades o comunidades deben contar con espacios que forman parte de su equipamiento urbano y que, en casos de emergencias, causadas por eventos de origen natural, o por el hombre, puedan cambiar su uso interno de manera que preste un servicio óptimo.

En el cantón Pedernales no hay un área deportiva organizada y que preste los servicios y comodidad a los conciudadanos que gusten o requieran de un espacio para hacer sus prácticas deportivas.

Tomando en cuenta que en el terremoto del $16 \mathrm{~A}$, no hubo un espacio adecuado para implantar albergues temporales que den posada emergente a las personas que perdieron sus viviendas o que corren peligro de colapsar, se plantea la necesidad de implantar un polideportivo en el cantón Pedernales en una ubicación seleccionada de acuerdo a las facilidades que debe prestar dando seguridad y de fáciles accesos peatonales y vehiculares desde el interior de la ciudad y exterior de otras comunidades cercanas, espacio que estaría equipado para ser utilizado en caso de emergencias con una capacidad de hospedaje para 1200 personas.

Un polideportivo puede cambiar su uso sin ser afectado su diseño arquitectónico y menos aún su equipamiento, es decir un área de canchas con techado puede ser utilizada para colocarse viviendas temporales (carpas), una sala de prensa como comedor temporal, los consultorios de odontología y medicina general seguirían prestando sus servicios dentro del áreas de salud, la 
administración no cambiaría su uso por cuanto es prioritario que alguna institución toma la dirección para el buen desempeño del uso temporal, las áreas verdes servirían como zona de recreación, de lectura de manualidades entre otros, el ingreso servirá igual para llevar un control adecuado de todo lo que sale y entra, las baterías higiénicas estarían funcionando igual para cualquier de los usos que tenga el polideportivo, la oficina del nutricionista servirá para direccionar el tipo de alimentación, en ambos casos estos espacios requieren de grandes almacenamientos del líquido vital, de almacenamiento de desechos, y de un aseo interior permanente. Sabiendo que terminada la emergencia este espacio volverá a prestar los servicios para lo que fue originalmente planificado.

\section{Plan de acción}

Con el presente plan de acción se procura direccionar las gestiones administrativas y gubernamentales en la gestión de residuos sólidos en albergues, a través de una estrategia coordinada y teniendo en cuenta distintos escenarios. Este procedimiento fue perfilado y relacionado de conformidad con los lineamientos instituidos en el régimen de vida de un albergue, tanto para el mejoramiento de la calidad de vida de los albergados como para la explotación eficiente de los residuos y siguiendo lo planteado en el actual reglamento ambiental del Ecuador.

El documento esboza un grupo de acciones dirigidas a desplegar una gestión procedente en la administración de los residuos sólidos, con el objetivo de mitigar los impactos generados debido a esta actividad en cada una de los espacios que componen los albergues, haciendo énfasis en las carpas.

\section{Lineamientos estratégicos}

Los lineamientos estratégicos de este plan de acción facilitarán la realización de operaciones articuladas que proporcionará el logro de los objetivos generales.

Para su planteamiento se han dividido en tres secciones: capacidades, institucionalidad e inversión, mediante las cuales, se presentan soluciones concretas y sostenibles que respondan a una apropiada gestión de los residuos sólidos en los albergues temporales

a. Líneas de acción de mejoramiento de capacidades 
1. Gestión local sostenible.

El fortalecimiento de los vínculos con instituciones de salud pública, educativas e investigativas en la búsqueda y desarrollo de tecnologías adecuada y de bajo costo para atender los problemas que genera la gestión de residuos sólidos.

\section{Producción limpia y responsabilidad social}

Se requiere de conocimientos, personal capacitado, servicios e infraestructuras apropiadas, fondos y capacidades técnicas. La responsabilidad social de tratar con seres humanos que se encuentran ante una compleja situación, no debe ser un impedimento para garantizar una gestión económica y ambientalmente sostenible.

b. Las líneas de acción de desarrollo de institucionalidad

- Fortalecimiento de la vigilancia sanitaria y ambiental

- Desarrollo de reglas orientadas a establecer parámetros para mejorar los procesos del manejo de residuos sólidos

- Potenciación de la transferencia tecnológica

- Desarrollo campañas educativas orientadas a cumplir las normas sobre higiene comunal

- Promoción de campañas de minimización y clasificación de residuos sólidos en la fuente

- Coordinación con las instituciones a distintos niveles (Municipio, Consejo Provincial, Secretaria Ambiental), para suscitar una cultura orientada a mejorar la calidad de vida a través de reducción de la contaminación ambiental y/o minimización de los residuos sólidos

c. Líneas de acción para la viabilidad de las inversiones: Promoción de la participación de instituciones privadas, gubernamentales y no gubernamentales en los programas de apoyo a los albergados y formulación y programación de las inversiones.

\section{Administración}

En situaciones de desastres naturales, la administración de los albergues corre a cargo del gobierno, quien delega los recursos en el Ministerio de Defensa y concretamente son las Fuerzas Armadas, quienes se encargan de organizar el funcionamiento de los procesos en estos enclaves 


\section{Financiamiento}

Las municipalidades habitualmente no poseen los recursos financieros para acometer proyectos en situaciones de desastre natural. Tampoco es común que dispongan de recursos adicionales para mejorar los procesos de higiene comunal y la gestión de residuos sólidos más allá de las actividades mínimas que hacen funcionar estos procesos. Por esa causa es necesario buscar alternativas de financiamiento para los proyectos que se incluyen en esta propuesta.

Dentro de esas alternativas puede estar la modificación de las partidas presupuestarias para instaurar, en el presupuesto anual de las localidades, partidas que incluyan los costos de los componentes del sistema propuesto, de modo que las entidades implicadas dispongan de recursos financieros para garantizar su funcionamiento.

También se puede valorar la posibilidad de obtener fondos a partir de la modificación de los sistemas contable y de cobro de los servicios del centro polideportivo, con el objetivo de obtener una fuente de ingresos que sirva para garantizar su sostenibilidad.

Otros actores importantes en la búsqueda de financiamiento son: las agencias de cooperación internacional, las organizaciones no gubernamentales y el sector privado.

Las oficinas de cooperación internacional, son instituciones comprometidas con el suministro de apoyo a los damnificados y a la gestión de residuos sólidos. Estas agencias pueden contribuir con equipamiento, recursos financieros y con colaboración técnica, y esto las transforma en importantes actores en casos de siniestros naturales. Como respuesta a esas intenciones y donaciones, las administraciones encargadas de la defensa civil se implican en este proceso, aportando personal, recursos materiales y capitales.

Los recursos financieros habitualmente son aprobados por el gobierno central hacia las administraciones municipalidades mediante acuerdos bilaterales. En ciertas situaciones estos acuerdos se firman directamente con las administraciones municipales, previo visto bueno del gobierno central.

Las organizaciones no gubernamentales sin fines de lucro con bastante frecuencia maniobran entre los sectores público y privado. A estas se les permite colaborar con las administraciones municipales, alcaldías o con las comunidades. Normalmente suelen contribuir con material 
técnico y soporte financiero y, del mismo modo que las oficinas de cooperación internacional están implicadas con el desarrollo de programas de atención afectados por fenómenos naturales y con el manejo integral de residuos sólidos. Es por esa razón, que tanto, las administraciones municipales como las alcaldías, deben buscar asociarse con las organizaciones no gubernamentales, a través de acuerdos de colaboración o convenios marco.

Existen varios modos en que el sector privado puede formar parte del apoyo que se realiza a las personas que son albergadas y en la propia gestión de residuos sólidos. Se debe tratar de empoderar a esta parte de la población con estas ideas, de modo que su aporte sea efectivo.

\section{Presupuesto}

El presupuesto se detalla para cada uno de los programas propuestos, tal como se muestra a continuación.

Tabla 3. Programa de capacitación y educación ambiental

\begin{tabular}{|c|c|c|c|c|c|c|}
\hline \multirow{2}{*}{ ACTIVIDAD } & \multicolumn{2}{|c|}{ RECURSOS } & \multirow{2}{*}{$\begin{array}{l}\text { Unidad de } \\
\text { medida }\end{array}$} & \multirow{2}{*}{$\begin{array}{c}\text { Cantidad } \\
{[\mathrm{u}]}\end{array}$} & \multirow{2}{*}{$\begin{array}{c}\text { Precio } \\
\text { unitario[ }[\$ / \mathrm{u}]\end{array}$} & \multirow{2}{*}{$\begin{array}{l}\text { Precio total } \\
{[\$]}\end{array}$} \\
\hline & Humano & Material & & & & \\
\hline \multirow{3}{*}{$\begin{array}{l}\text { CHARLAS EN los predios del } \\
\text { albergue } \\
\text { (alumnos y docentes) }\end{array}$} & $\mathrm{X}$ & & horas & 30,00 & 10,00 & 300,00 \\
\hline & & Proyector & unidad & 1,00 & 650,00 & 650,00 \\
\hline & & Manuales & unidad & 3200,00 & 0,15 & 480,00 \\
\hline \multirow{2}{*}{$\begin{array}{l}\text { Orientaciones y rendiciones de } \\
\text { cuentas de ENTIDADES } \\
\text { PÚBLICAS encargadas de la } \\
\text { gestión de residuos sólidos }\end{array}$} & $\mathrm{X}$ & & horas & 30,00 & 10,00 & 300,00 \\
\hline & & Manuales & unidad & 3200,00 & 0,15 & 480,00 \\
\hline & & & & \multicolumn{2}{|c|}{ SUBTOTAL [\$] } & 2210,00 \\
\hline & & & & \multicolumn{2}{|c|}{ Imprevistos 5\% [\$] } & 110,50 \\
\hline & & & & \multicolumn{2}{|r|}{ TOTAL [\$] } & 2320,50 \\
\hline
\end{tabular}

Fuente: El autor, 2017

Tabla 4. Programa de separación de residuos sólidos en el origen

\begin{tabular}{|c|c|c|c|c|c|c|}
\hline \multirow{2}{*}{ ACTIVIDAD } & \multicolumn{2}{|c|}{ RECURSOS } & \multirow{2}{*}{$\begin{array}{l}\text { Unidad de } \\
\text { medida }\end{array}$} & \multirow{2}{*}{$\begin{array}{c}\text { Cantidad } \\
{[\mathrm{u}]}\end{array}$} & \multirow{2}{*}{$\begin{array}{c}\text { Precio } \\
\text { unitario[ } \$ / \mathrm{u}]\end{array}$} & \multirow{2}{*}{$\begin{array}{l}\text { Precio total } \\
{[\$]}\end{array}$} \\
\hline & Humano & Material & & & & \\
\hline $\begin{array}{l}\text { Fortalecer la separación de } \\
\text { residuos en la fuente }\end{array}$ & & Recipientes & unidad & 1800 & 3,00 & 5400,00 \\
\hline & & & & \multicolumn{2}{|c|}{ SUBTOTAL } & 5400,00 \\
\hline & & & & \multicolumn{2}{|c|}{ Imprevistos 5\% } & 270,00 \\
\hline & & & & \multicolumn{2}{|c|}{ TOTAL } & 5670,00 \\
\hline
\end{tabular}

Fuente: El autor, 2017 
Tabla 5. Programa de higiene interior del albergue

\begin{tabular}{|c|c|c|c|c|c|c|}
\hline \multirow[b]{2}{*}{ ACTIVIDAD } & \multicolumn{2}{|c|}{ RECURSOS } & \multirow{2}{*}{$\begin{array}{c}\text { Unidad } \\
\text { de } \\
\text { medida }\end{array}$} & \multirow{2}{*}{$\begin{array}{c}\text { Cantidad } \\
{[\mathrm{u}]}\end{array}$} & \multirow{2}{*}{$\begin{array}{c}\text { Precio } \\
\text { unitario } \\
{[\$ / \mathrm{u}]}\end{array}$} & \multirow{2}{*}{$\begin{array}{c}\text { Precio } \\
\text { total } \\
{[\$]} \\
\end{array}$} \\
\hline & Humano & Material & & & & \\
\hline $\begin{array}{l}\text { Fortalecer la separación de residuos } \\
\text { en los puntos de recogida }\end{array}$ & & Tanques & unidad & 12 & 25,00 & 300,00 \\
\hline \multirow{2}{*}{ Mantenimiento de la higiene } & & $\begin{array}{c}\text { Set de } \\
\text { herramientas }\end{array}$ & unidad & 2 & 15,00 & 30,00 \\
\hline & & $\begin{array}{l}\text { Uniforme e } \\
\text { indumentaria }\end{array}$ & unidad & 2 & 45,00 & 90,00 \\
\hline & & & & \multicolumn{2}{|c|}{ SUBTOTAL } & 420,00 \\
\hline & & & & \multicolumn{2}{|c|}{ Imprevistos 5\% } & 21,00 \\
\hline & & & & \multicolumn{2}{|c|}{ TOTAL } & 441,00 \\
\hline
\end{tabular}

Fuente: El autor, 2017

Tabla 6. Programa de creación de un área urbana que pueda emplearse como área deportiva y como albergue temporal

\begin{tabular}{|c|c|c|c|c|}
\hline Rubro & \begin{tabular}{|l|} 
Unidad de \\
medida \\
\end{tabular} & $\begin{array}{l}\text { Cantidad } \\
{[\mathrm{u}]} \\
\end{array}$ & $\begin{array}{l}\text { Precio } \\
\text { unitario }[\$ / \mathrm{u}]\end{array}$ & Precio total $[\$]$ \\
\hline Ingreso & $\mathrm{m}^{2}$ & 60 & 350,00 & $21.000,00$ \\
\hline Información & $\mathrm{m}^{2}$ & 24 & 350,00 & $8.400,00$ \\
\hline Administración & $\mathrm{m}^{2}$ & 260 & 350,00 & $91.000,00$ \\
\hline Salón de uso múltiples & $\mathrm{m}^{2}$ & 340 & 350,00 & $119.000,00$ \\
\hline Área de prensa & $\mathrm{m}^{2}$ & 80 & 350,00 & $28.000,00$ \\
\hline Medicina general & $\mathrm{m}^{2}$ & 40 & 350,00 & $14.000,00$ \\
\hline Nutricionista & $\mathrm{m}^{2}$ & 20 & 350,00 & $7.000,00$ \\
\hline Gimnasio & $\mathrm{m}^{2}$ & 240 & 350,00 & $84.000,00$ \\
\hline Bodega & $\mathrm{m}^{2}$ & 80 & 350,00 & $28.000,00$ \\
\hline $\begin{array}{c}\text { Canchas de uso múltiples (6u) } \\
\text { incluida su cubierta }\end{array}$ & $\mathrm{m}^{2}$ & 5700 & 120,00 & $684.000,00$ \\
\hline $\begin{array}{l}\text { Canchas de futbol con césped } \\
\text { sintético }(2 \mathrm{u})\end{array}$ & $\mathrm{m}^{2}$ & 8100 & 90,00 & $729.000,00$ \\
\hline Áreas verdes & $\mathrm{m}^{2}$ & 15256 & 10,00 & $152.560,00$ \\
\hline & & & SUBTOTAL & 1'965.960,00 \\
\hline & & & $12 \%$ IVA & $235.915,20$ \\
\hline & & & TOTAL & $2 ’ 201.875,20$ \\
\hline
\end{tabular}

Fuente: El autor, 2017

\section{Evaluación}

Se propone una herramienta que permite calificar los sitios que fungen o que podrían potencialmente fungir como albergues temporales, teniendo en cuenta aspectos urbanísticos, climáticos, espaciales y geotécnicos. Esta herramienta, ha sido establecida a partir del consenso 
de expertos en la temática, quienes han ponderado cada una de las variables e indicadores empleados, basada en una evaluación multicriterio. La herramienta fue empleada, tanto para valorar los sitios utilizados para albergues, como para juzgar la conveniencia de emplear un área citadina que desempeñe la función de centro polideportivo en situaciones convencionales y de albergue temporal, ante una catástrofe natural.

Para realizar una valoración del plan de gestión de los residuos sólidos de carácter domiciliar, producidos por los desplazados internos de los albergues del cantón Pedernales, se debe tener presente que este nació de las deficiencias observadas en el funcionamiento de dicho manejo en los albergues "Divino Niño" y "Los Llanos" de Pedernales. En consecuencia, se decide elaborar un documento, que recoge información actualizada y fidedigna sobre este escenario, con el fin de mejorar y reorientar las planificaciones estratégicas, en aras de corregir estas actividades a través de un trabajo social, económica y ambientalmente sostenible. El programa está orientado hacia a la salud pública y a la promoción de una mejor calidad de vida de los desplazados internos.

\section{Referencias Bibliográficas}

Abreu Guirado, O., Rodríguez Heredia, O., Pérez Delgado, E., \& González García, M. (2008). Bioseguridad: su comportamiento. Revista Archivo Médico de Camagüey, 12(5), 0-0.

CRED. (2015). Poverty and Death: Disaster Mortality 1996-2015. Centre for Research on the Epidemiolgy of Disaster. (CRED). The United Nations Office for Disaster Risk Reduction. Disponible en: http://cred.be/sites/default/files/CRED_Disaster_Mortality.pdf

Diario Oficial de las Comunidades Europeas (1991). Directiva del Consejo 75/442/CEE, relativa a los residuos (con las modificaciones de la Directiva del Consejo 91/156/CEE de 18 de marzo de 1991). Diario Oficial de las Comunidades Europeas, (15).

Glyn Jun. Escalona Héctor. 1991. Ingeniería ambiental, Residuos sólidos, Ed. Pearson educación. España.568-572 pg. 36,37 pg.

Landeta, J. (1999). El método Delphi. Una técnica de previsión del futuro. Ariel, Landeta, J. (1999). El método Delphi. Una técnica de previsión del futuro. 
Ministerio del ambiente. (2014). "Estudio para conocer los potenciales impactos ambientales y vulnerabilidad relacionada con las sustancias químicas y tratamiento de desechos peligrosos en el sector productivo de Ecuador. Quito: Gobierno Nacional del Ecuador.

OMS. (2015). Temas de salud. Saneamiento. Obtenido de http://www.who.int/topics/sanitation/es/

Proyecto Esfera. (2007). El Proyecto Esfera. 10 años de Esfera en Acción: Mejorar la calidad y la rendición de cuentas en la acción humanitaria 1997-2007. Sociedad Nacional de La Cruz Roja Colombiana (2008). Manual Nacional para el manejo de Albergues Temporales. Dirección General del Socorro Nacional. Disponible en: http://web.cruzrojacolombiana.org/publicaciones/pdf/manual_final_albergues_temporales_19120 11_035711.pdf

Wehenpohl, G., Hernández Barrios, C. P., Rodriguez Salinas, M. A., y Escudero Quijano, I. (2006). Guía para la elaboración de programas municipales para la prevención y gestión integral de los residuos sólidos urbanos. In Guía para la elaboración de programas municipales para la prevención y gestión integral de los residuos sólidos urbanos. SEMARNAT/GTZ. 